\title{
INTERNATIONAL AFIR COLLOQUIUM 2003
}

\section{Wednesday 17 - Friday 19 September 2003}

MECC - Maastricht - The Netherlands

Registration is still possible

You will find the registration form on our website www.afir2003.nl

Hotel Accommodation

We have blocked the rooms until 17 July 2003.

If you want first choice book early with the hotelreservation form on www.afir2003.nl

More information

Visit our website www.afir2003.nl for up-to-date information.

\section{See you in Maastricht!}

Organising Committee

Hugo Berkouwer (Chairman), Pari Kandhai, Marcel Thijssen, Robert de Vries, Inge Huizinga

Scientific Committee

Ruud Kleynen (Chairman), Angela van Heerwaarden (Vice-Chairman), Guus Boender, Marc de Ceuster, Michael Damm, Marc Goovaerts, Steven Haberman, Antoon Koolen, Alfred Oosenbrug, Peter Schotman and Rolf Tschernig

AFIR 2003 Secretariat

Het Actuarieel Genootschap, att. Inge Huizinga, Postbus 540, 3440 AM Woerden, The Netherlands tel: + 313484396 23; fax: + 313484305 55; e-mail: inge.huizinga@ag-ai.nl 